\title{
Diversity and distribution of epiphytic lichens and bryophytes on aspen (Populus tremula) in the middle boreal forests of Republic of Karelia (Russia)
}

\author{
Viktoria N. Tarasova ${ }^{1}$, Roman P. Obabko ${ }^{1}$, Dmitry E. Himelbrant ${ }^{2,3}$, \\ Margarita A. Boychuk ${ }^{4}$, Irina S. Stepanchikova ${ }^{2,3} \&$ Eugene A. Borovichev $^{5,6}$ \\ ${ }^{1}$ Department of Botany and Plant Physiology, Petrozavodsk State University, 33 Lenin St., 185910 Petrozavodsk, Russia. \\ E-mails: vika18@sampo.ru, romaparrot@mail.ru \\ ${ }^{2}$ St. Petersburg State University (SPbSU), 7-9 Universitetskaya Emb., 199034 St. Petersburg, Russia \\ ${ }^{3}$ Laboratory of Lichenology and Bryology, Komarov Botanical Institute RAS, 2 Professor Popov St., 197376 St. \\ Petersburg, Russia.E-mails: stepa_ir@mail.ru, d_brant@mail.ru \\ ${ }^{4}$ Laboratory of Mire Ecosystems, Institute of Biology of Karelian Research Centre of RAS, 11 Pushkinskaya St., 185910 \\ Petrozavodsk, Russia. E-mail: boychuk@krc.karelia.ru \\ ${ }^{5}$ Laboratory of Terrestrial Ecosystems, Institute of the Industrial Ecology Problems of the North of the Kola Science \\ Centre RAS, Academic Campus, 14a, Apatity, Murmansk Region, 184209, Russia. E-mail: borovichyok@mail.ru \\ ${ }^{6}$ Laboratory for Boreal Forest Dynamics and Production, Forest Research Institute, Karelian Research Centre RAS, \\ Petrozavodsk, 185910, Russia
}

\begin{abstract}
The distribution of epiphytic bryophyte and lichen species growing on aspen in the middle boreal forests was studied in southern Karelia (Russia). These forests varied in time-since-disturbance from 80 to 450 years. Two hundred twenty two species of epiphytes, including 178 lichens, 32 mosses and 12 liverworts, were recorded on 192 aspen trees in forests over 24 ha, in the Karelian part of the Vodlozero National Park, Kivach Strict Nature Reserve, Kizhi Sanctuary and Petrozavodsk City. Arthonia biatoricola, A. excipienda and Biatoridium monasteriense were collected in Karelia for the first time. Eighteen rare species (lichens Anaptychia ciliaris, Arthonia vinosa, Bryoria nadvornikiana, Chaenotheca gracilenta, C. stemonea, Lecidea albofuscescens, Lobaria pulmonaria, Melanelixia subaurifera, Nephroma bellum, N. laevigatum, Phaeocalicium populneum, Ramalina thrausta, Rostania occultata, Scytinium subtile, Usnea barbata, mosses Neckera pennata, Plagiomnium drummondii and liverwort Lejeunea cavifolia) listed in the Red Data Book of Republic of Karelia (2007) were found. Relationships between epiphytic lichen and bryophyte species richness and certain environmental variables (at different trunk heights above ground and time-since-disturbance) were evaluated. Lichens and mosses on aspen trunks often occupy different ecological niches. Cover and diversity of bryophytes was high on trunk bases, while the number of lichen species and their cover were higher at a height of $1.3 \mathrm{~m}$ above ground level. The total number of lichen species on aspen increased on average from 40 to 60 species per ha with increasing time-since-disturbance from 100 to 450 years. A stabilization in lichen species number was observed at about 200 years since disturbance. No significant correlation was determined between bryophyte diversity on aspens and the time-since-disturbance.
\end{abstract}

Keywords: epiphytes on aspen; middle boreal forests; lichens; bryophytes; liverworts; time-since-disturbance

\section{INTRODUCTION}

Epiphytic lichens and bryophytes are important components of many forest ecosystems. They exhibit high species diversity, form conspicuous biomass and play a significant ecological role in boreal ecosystems (Esseen et al., 1996; Glime, 2007). In addition, lichen epiphytes are useful indicators of forest health because they are sensitive to forest management practices and serve as indicators of air quality (Lesica et al., 1991; Richardson, 1992; McCune, 2000). According to literature, the most important factors influencing epiphytic bryophyte and lichen distribution are forest stand age (Fritz et al., 2008; Mežaka et al., 2010), host tree (Lõhmus et al., 2007; Mežaka et al., 2008; Strazdina, 2010), tree age (Barkman, 1958; Hedenås \& Eriksson, 2000; Ojala et al., 2000) and microclimate (John \& Dale, 1995).

Aspen (Populus tremula L.) is a common deciduous tree species in the middle and south boreal taiga, which can form secondary forests (pure aspen stands and mixed aspen-spruce stands). Aspen also occurs in mesic stands in 
old-growth spruce forests by renewal in canopy gaps due to natural gap dynamics (Kuusinen, 1994; Hazell et al., 1998). It reproduces mainly vegetatively by root suckers. Mature aspen trees (their trunks and roots) are frequently attacked by several pathogenic fungi, for example by Phellinus tremulae (Bondartsev) Bondartsev \& P. N. Borisov (Kuusinen, 1994). In spite of this, Populus trees often reach an age of 200 years in Scandinavian forests (Kuusinen, 1994). Aspen trees in suitable habitats usually grow very quickly, and may reach large proportions - tree height up to $40 \mathrm{~m}$, diameter at breast height (DBN) up to $1 \mathrm{~m}$ - even when relatively young (Abaimov, 2009). Angles between branches and trunk of aspen are sharp (acute), therefore most rain water intercepted by the crown flows down the tree trunks. High values of tree height, crown length and radius increase stemflow, which in aspen can reach $9 \%$ of the total precipitation in the community (Molchanov, 1961). In addition, the aspen tree usually does not shed its bark. Aspen has a rather nutrient-rich bark with a relatively high $\mathrm{pH}(5-7)$ and moisture capacity (Barkman, 1958; Gustafsson \& Eriksson, 1995). Therefore, aspen provides an important substrate for many epiphytes (Kuusinen, 1994; Gustafsson \& Eriksson, 1995), including lichens, bryophytes and liverworts.

Epiphyte diversity on Populus tremula in boreal forests in Europe has been intensively studied (Shubina et al., 1996; Uliczka \& Angelstam, 1999; Lõhmus, 2003). Many researches focused on studying aspen as an important component in preserving biodiversity in boreal forests, because large numbers of specialist species are entirely dependent on this species (Kuusinen \& Siitonen, 1998; Pykälä et al., 2006). There were the studies on cyanolichens and lichens associated with Lobaria pulmonaria (L.) Hoffm. on aspen, which is the one of main host trees for these lichens in boreal forests in Europe (Gauslaa, 1995; Kuusinen, 1996; Gjerde et al., 2012). However, the epiphytic lichen and bryophyte diversity of aspen in northwest Russia is still poorly studied (Pystina \& Hermansson, 1996; Mikhailova et al., 2005). The main aim of the current research was to inventory the lichen and bryophyte diversity associated with aspen trees in the middle boreal forests of Republic of Karelia.

\section{MATERIALS AND METHODS}

\section{Study areas}

Study was carried out during summer 2014 and 2015 in mesic, middle boreal forests in the southern part of the Republic of Karelia, Russia. The climate is predominantly temperate and intermediate ranging from oceanic to continental and characterized by having relatively mild, long winters and cool, short summers. The mean annual temperature is $+3^{\circ} \mathrm{C}$ and the mean annual precipitation is $450-750 \mathrm{~mm}$ (Nazarova, 2003). The area is flat, varying not more than $100 \mathrm{~m}$ in elevation. Low humic podzolic soils predominate in not waterlogged habitats (Morozova, 1991). Forests cover more than $54 \%$ of the Karelian territory. Pine communities predominate $(64 \%$ of the forested area), while spruce communities occupy $25 \%$ of the area (Volkov, 2008). Over the past 80 years, the area of small-leaved secondary forests in Karelia has tripled due to intensive forest management and now they occupy $11 \%$ of the forested territory. Aspen stands occupy $4.2 \%$ of the forested territory in the middle taiga (Volkov, 2008).

Research was carried out in four study areas: the Karelian part of the Vodlozero National Park, Kivach Strict Nature Reserve, Kizhi Sanctuary and Petrozavodsk City (Fig. 1). In each territory, permanent sample plots of one ha $(100 \times 100$ $\mathrm{m})$ were established in communities at different successional stages, belonging to the same ecological-dynamic series. These communities represent the process of restoration of climax spruce forest of Vaccinium myrtillus - green mosses type, occupying plains on loamy moraine, without stagnant moisture. In general, spruce forests after fire or clear cuttings regenerate through different early serial stages, usually involving birch or aspen (Kazimirov, 1971; Dyrenkov, 1984), with aspen on richer soils and birch on poorer ones (Ipatov, 1960; Degteva et al., 2001). In this study, the successional dynamics of spruce forests at different successional stages varying in time-since-disturbance from 80 to 450 years is considered (Table 1). Based on literature data of successions in the mid-taiga spruce forests (Kazimirov, 1971; Dyrenkov, 1984), all studied communities correspond to four groups according to different stages of the ecological-dynamic series: 


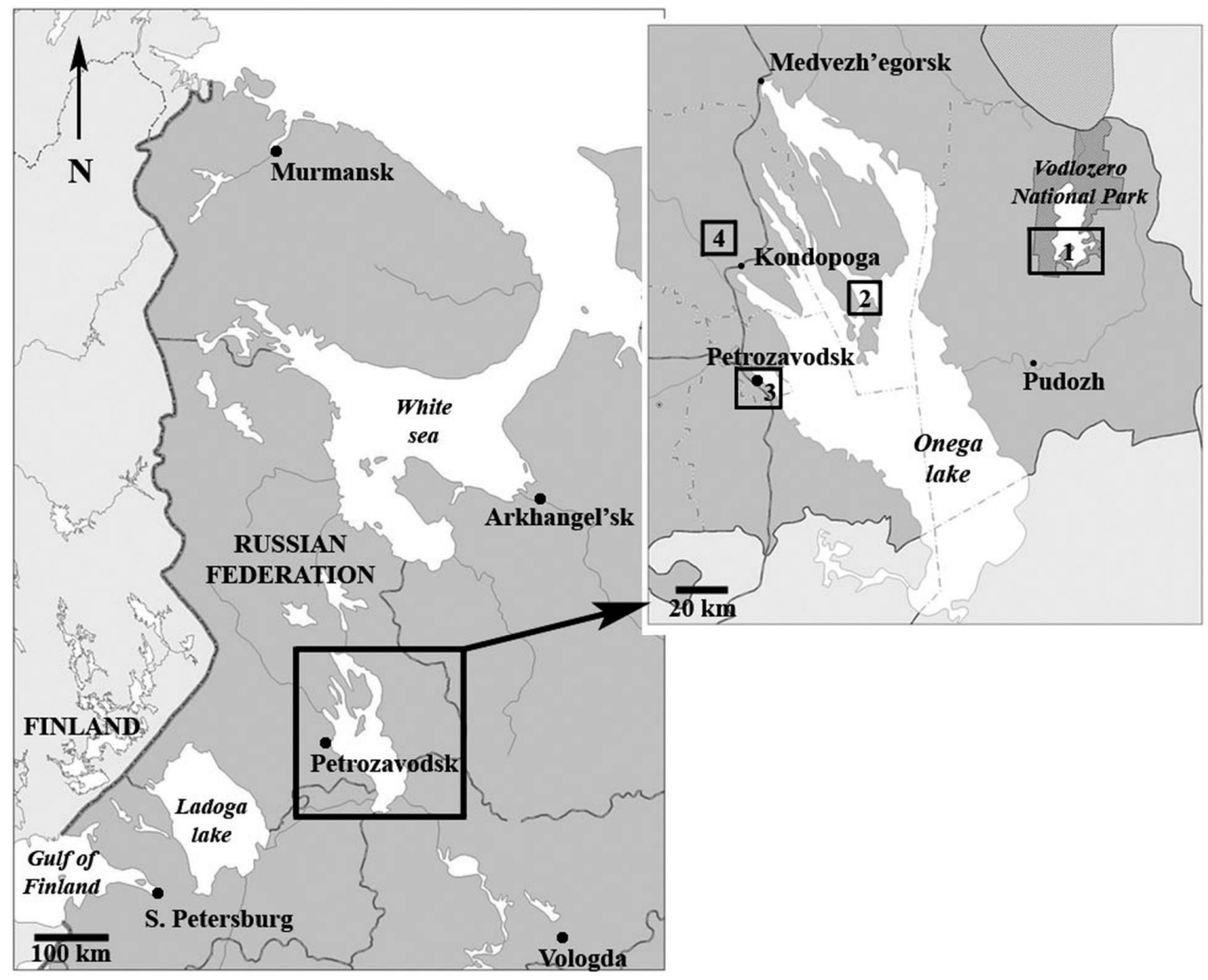

Fig. 1. The location of the study areas (Republic of Karelia, NW Russia): 1 - Vodlozero National Park, 2 - Kizhi Sanctuary, 3 - Kivach Strict Nature Reserve, 4 - Petrozavodsk City.

1) middle-aged aspen forest Calamagrostis arundinacea-Vaccinium myrtillus type; 2) mixed aspen-spruce forest Vaccinium myrtillus-Calamagrostis arundinacea type; 3) pre-climax spruce forest Vaccinium myrtillus-green mosses type; 4) climax (old-growth) spruce forest Vaccinium myrtillus-green mosses type (Table 1). In each study area, forests communities from the first three stages of succession were represented, while all successional stages of spruce forest were studied in the Vodlozero National Park only. The main characteristics of the studied forest communities on 24 sample plots are given in Table 1.

\section{Data collection}

To estimate time-since-disturbance in the studied communities, the population structure of each tree stand (mainly spruce trees) was evaluated following Stavrova et al. (2016). This method is described in detail in Ignatenko \& Tarasova (2017). Eight aspen trees were randomly chosen within each sample plot to assess epiphytic biodiversity (lichens, lichenicolous and non-lichenized fungi, mosses and liverworts). Species diversity and cover of lichens, mosses and liverworts were estimated by means of a $25 \times$ $25 \mathrm{~cm}$ frame at the northern, southern, eastern and western sides of a trunk at the base and breast height (at $1.3 \mathrm{~m}$ from base). In addition, species diversity was recorded on branches of recently fallen aspen trees within each sample plot. In total, 1535 descriptions of the epiphytic cover were made on 192 aspen trees from 24 sample plots (Vodlozero National Park - 7 sample plots, Kizhi Sanctuary - 6, Kivach Strict Nature Reserve - 8, Petrozavodsk City - 3). 
Table 1. Main characteristics of studied forest communities in the middle taiga of Republic of Karelia. Study areas, 1 - Vodlozero National Park, 2 - Kizhi Sanctuary, 3 - Kivach Strict Nature Reserve, 4 - Petrozavodsk City; type of forests, a - middle-aged aspen forest Vaccinium myrtillusCalamagrostis arundinacea type, a-s - mixed aspen-spruce forest Calamagrostis arundinacea-Vaccinium myrtillus type, spc - pre-climax spruce forest Vaccinium myrtillus-green mosses type, sc - climax (old-growth) spruce forest Vaccinium myrtillus-green mosses type.

\begin{tabular}{|c|c|c|c|c|c|c|c|c|c|}
\hline \multirow[t]{2}{*}{$\begin{array}{l}\text { Study } \\
\text { area }\end{array}$} & \multirow{2}{*}{$\begin{array}{c}\text { No of } \\
\text { sample } \\
\text { plot }\end{array}$} & \multirow[t]{2}{*}{ GPS coordinate } & \multirow[t]{2}{*}{$\begin{array}{l}\text { Type of } \\
\text { forest }\end{array}$} & \multirow{2}{*}{$\begin{array}{c}\text { Time-since- } \\
\text { disturbance, } \\
\text { years }\end{array}$} & \multirow[t]{2}{*}{$\begin{array}{c}\text { Basal area, } \\
\mathrm{m}^{2} \mathrm{ha}^{-1}\end{array}$} & \multicolumn{2}{|c|}{$\begin{array}{l}\text { Proportion in the } \\
\text { tree stand, \% }\end{array}$} & \multicolumn{2}{|c|}{ Tree age, years } \\
\hline & & & & & & Spruce & Aspen & Spruce & Aspen \\
\hline \multirow[t]{7}{*}{1} & 1 & $62^{\circ} 13.448^{\prime} \mathrm{N}, 36^{\circ} 45.222^{\prime} \mathrm{E}$ & spc & 260 & 19.3 & 64 & 2 & $46-229$ & $128-152$ \\
\hline & 2 & $62^{\circ} 12.001^{\prime} \mathrm{N}, 36^{\circ} 51.114^{\prime} \mathrm{E}$ & a & 80 & 29 & 8 & 33 & $28-48$ & $26-73$ \\
\hline & 3 & $62^{\circ} 12.076^{\prime} \mathrm{N}, 36^{\circ} 50.249^{\prime} \mathrm{E}$ & $a-s$ & 180 & 31 & 65 & 14 & $48-143$ & $67-151$ \\
\hline & 4 & $62^{\circ} 11.972^{\prime} \mathrm{N}, 36^{\circ} 51.009^{\prime} \mathrm{E}$ & $a-s$ & 160 & 26.5 & 53 & 22 & $28-84$ & $83-151$ \\
\hline & 5 & $62^{\circ} 11.976^{\prime} \mathrm{N}, 36^{\circ} 49.659^{\prime} \mathrm{E}$ & a & 100 & 30.3 & 27 & 45 & $25-95$ & $56-103$ \\
\hline & 6 & $62^{\circ} 13.545^{\prime} \mathrm{N}, 37^{\circ} 05.081^{\prime} \mathrm{E}$ & sc & 410 & 22.8 & 81 & 12 & $24-263$ & $164-198$ \\
\hline & 7 & $62^{\circ} 13.026^{\prime} \mathrm{N}, 37^{\circ} 03.456^{\prime} \mathrm{E}$ & sc & 450 & 29.3 & 89 & 9 & $31-180$ & 109-203 \\
\hline \multirow[t]{6}{*}{2} & 1 & $62^{\circ} 06.727^{\prime} \mathrm{N}, 35^{\circ} 09.241^{\prime} \mathrm{E}$ & $a-s$ & 160 & 30.5 & 42 & 35 & $61-122$ & $84-132$ \\
\hline & 2 & $62^{\circ} 06.619^{\prime} \mathrm{N}, 35^{\circ} 09.353^{\prime} \mathrm{E}$ & $a-s$ & 150 & 31 & 51 & 29 & $42-126$ & $71-130$ \\
\hline & 3 & $62^{\circ} 07.057^{\prime} \mathrm{N}, 36^{\circ} 07.267^{\prime} \mathrm{E}$ & $\mathrm{spc}$ & 260 & 22 & 81 & 13 & $49-192$ & $81-169$ \\
\hline & 4 & $62^{\circ} 06.897^{\prime} \mathrm{N}, 35^{\circ} 07.252^{\prime} \mathrm{E}$ & spc & 240 & 28 & 73 & 12 & $22-145$ & 88-198 \\
\hline & 5 & $62^{\circ} 06.843^{\prime} \mathrm{N}, 35^{\circ} 09.747^{\prime} \mathrm{E}$ & a & 85 & 24 & 1 & 60 & $36-55$ & $39-85$ \\
\hline & 6 & $62^{\circ} 06.902^{\prime} \mathrm{N}, 35^{\circ} 09.546^{\prime} \mathrm{E}$ & $\mathrm{a}$ & 100 & 19 & 5 & 68 & $36-75$ & 39-97 \\
\hline \multirow[t]{3}{*}{3} & 1 & $61^{\circ} 44.906^{\prime} \mathrm{N}, 34^{\circ} 21.906^{\prime} \mathrm{E}$ & $\mathrm{a}$ & 110 & 25 & 34 & 38 & $19-78$ & $57-104$ \\
\hline & 2 & $61^{\circ} 44.268^{\prime} \mathrm{N}, 34^{\circ} 19.202^{\prime} \mathrm{E}$ & $a-s$ & 160 & 32 & 44 & 43 & $38-150$ & $53-117$ \\
\hline & 3 & $61^{\circ} 50.472^{\prime} \mathrm{N}, 34^{\circ} 30.482^{\prime} \mathrm{E}$ & spc & 210 & 28 & 59 & 22 & $84-168$ & $87-119$ \\
\hline \multirow[t]{8}{*}{4} & 1 & $62^{\circ} 16.678^{\prime} \mathrm{N}, 33^{\circ} 59.135^{\prime} \mathrm{E}$ & $a-s$ & 170 & 27 & 61 & 22 & $30-147$ & $73-163$ \\
\hline & 2 & $62^{\circ} 16.599^{\prime} \mathrm{N}, 33^{\circ} 59.706^{\prime} \mathrm{E}$ & $a-s$ & 160 & 31 & 38 & 38 & $46-126$ & $72-115$ \\
\hline & 3 & $62^{\circ} 16.517^{\prime} \mathrm{N}, 33^{\circ} 59.828^{\prime} \mathrm{E}$ & $a-s$ & 170 & 37 & 45 & 44 & $28-82$ & $63-169$ \\
\hline & 4 & $62^{\circ} 15.794^{\prime} \mathrm{N}, 33^{\circ} 58.855^{\prime} \mathrm{E}$ & a & 80 & 39 & 30 & 68 & $31-72$ & $42-80$ \\
\hline & 5 & $62^{\circ} 15.879^{\prime} \mathrm{N}, 33^{\circ} 58.730^{\prime} \mathrm{E}$ & $a-s$ & 190 & 23.5 & 46 & 35 & $21-153$ & $47-161$ \\
\hline & 6 & $62^{\circ} 15.997^{\prime} \mathrm{N}, 33^{\circ} 59.685^{\prime} \mathrm{E}$ & $a-s$ & 170 & 33.5 & 53 & 32 & $21-132$ & $102-169$ \\
\hline & 7 & $62^{\circ} 17.051^{\prime} \mathrm{N}, 33^{\circ} 58.271^{\prime} \mathrm{E}$ & spc & 240 & 21 & 74 & 13 & $24-159$ & $97-186$ \\
\hline & 8 & $62^{\circ} 17.225^{\prime} \mathrm{N}, 33^{\circ} 57.817^{\prime} \mathrm{E}$ & $\mathrm{spc}$ & 250 & 26 & 77 & 12 & $21-211$ & $92-156$ \\
\hline
\end{tabular}

Collected material was identified in the Herbarium of the Botanical Museum, Finnish Museum of Natural History, University of Helsinki; Department of Botany and Plant Physiology, Petrozavodsk State University; Department of Botany, St. Petersburg State University; Laboratory of Mire Ecosystems, Institute of Biology of Karelian Research Centre RAS, Petrozavodsk; Laboratory of Terrestrial Ecosystems, Institute of the Industrial Ecology Problems of the North of the Kola Science Centre RAS, Apatity and Laboratory for Boreal Forest Dynamics and Production, Forestry Research Institute of Karelian Research Centre RAS, Petrozavodsk. Selected specimens from genus Cladonia and sterile crustose lichens were identified by a standard technique of thin-layer chromatography (TLC) in the Laboratory of Experimental Botany of Petrozavodsk State University, Petrozavodsk, using solvent systems A, B and C (Orange et al., 2001). In total, 400 specimens of mosses and liverworts and 3447 specimens of lichensand allied fungi were identified; 188 specimens were identified using TLC. Representative specimens were deposited in the herbarium of Petrozavodsk State University (PZV); doublet liverwort specimens were kept in the herbarium of the Institute of the Industrial Ecology Problems 
of the North of the Kola Science Centre RAS (INEP). Simple non-linear regression analysis was used to evaluate the relationships between species number (separately for lichens, mosses and liverworts) and the time-since-disturbance. Comparison between groups of the main characteristics of lichen and bryophyte distribution (cover and number of species) were tested by means of Mann-Whitney one-way analysis of variance. The program Statgraphics Centurion XV (2006) was used.

\section{List of localities}

The following study areas were investigated: KvR - Kivach Strict Nature Reserve $(10,450$ ha), Kondopoga District, $62^{\circ} 20^{\prime} \mathrm{N}, 34^{\circ} 00^{\prime} \mathrm{E}$, biogeographical province Karelia onegensis (Kon); Ptz - Petrozavodsk City (11300 ha), Prionezhsky District, $61^{\circ} 50^{\prime} \mathrm{N}, 34^{\circ} 20^{\prime} \mathrm{E}$, biogeographical provinces Karelia onegensis and Karelia olonetsensis (Kol); VNP - Karelian part of the Vodlozero National Park (130,600 ha), Pudozh district, $62^{\circ} 30^{\prime} \mathrm{N}, 36^{\circ} 55^{\prime} \mathrm{E}$, biogeographical province Karelia transonegensis (Kton); KzR - Kizhi Sanctuary (50,000 ha), Medvezh'egorsk District, Zaonezhsky Peninsula, 62 $12^{\prime} \mathrm{N}, 35^{\circ} 14^{\prime} \mathrm{E}$, biogeographical province Karelia onegensis.

\section{THE SPECIES}

Taxa are arranged in alphabetical order; nomenclature of lichens, lichenicolous and non-lichenized fungi follows mainly Nordin et al. (2011), nomenclature of liverworts mainly Söderström et al. (2016) with some updates from other literature (Konstantinova et al., 2009), and nomenclature of mosses - Hill et al. (2006).

Abbreviations and symbols: \# - lichenicolous fungi; + - non-lichenized fungi; ! - new species for Republic of Karelia; Kol! - new species for the biogeographical province Karelia olonetsensis, Kon!- new species for the biogeographical province Karelia onegensis, Kton! - new species for the biogeographical province Karelia transonegensis; KvR!, KzR!, Ptz!, VNP! - new species for study areas: KvR - Kivach Strict Nature Reserve, KzR - Kizhi Sanctuary, Ptz - Petrozavodsk City, VNP - Vodlozero National Park; other biogeographical provinces: $\mathrm{Kl}$-Karelia ladogensis, $\mathrm{Kk}$ - Karelia keretina, Ks - Regio kuusamoënsis. Species included in the Red Data Book of the Republic of Karelia (2007) are marked as RK, and in the Red Data Book of the Russian Federation (2008) - RR. The types of forest communities are marked with the numbers 1-4 (see Tab. 1). KzR!? - exact locality of this species, published by Norrlin (1876) for Zaonezhsky Peninsula (included the area of Kizhi Sanctuary) (Fadeeva et al., 2014), is not established due to old toponyms.

\section{a. Lichens, lichenicolous and non-lichenized fungi}

Acrocordia gemmata (Ach.) A. Massal. - KzR: 2. Kon! KzR!

AlyXORIA VARIA (Pers.) Ertz \& Tehler - KvR, KzR, VNP: 1-4. Kton! KzR!

Amandinea Punctata (Hoffm.) Coppins \& Scheid. - VNP: 2.

ANAPTYCHIA CILIARIS (L.) Körb. - Ptz: 2. RK.

ANISOMERIDIUM POLYPORI (Ellis \& Everh.) M. E. Barr - KvR, KzR, Ptz, VNP: 1-4. Kon! Kol! Kton! KvR! KzR! Ptz! VNP!

ARCTOMIA FASCICULARIS (L.) Otálora \& Wedin - KvR: 2; VNP: 4. This species was recently found in Karelia in provinces Kon and Kton (Tarasova \& Stepanchikova, 2016).

Arthonia apatetica (A. Massal.) Th. Fr. - KvR, Ptz: 1-3. Kon! KvR!

!\#ARTHONIA BIATORICOLA Ihlen \& Owe-Larss. - KvR: right bank of the Suna River, below Kivach waterfall, $62^{\circ} 15.879^{\prime} \mathrm{N}, 33^{\circ} 58.730$ ' $\mathrm{E}$, on thalli of Biatora efflorescens, 2,19.09.2015. Kon! KvR! This recently described lichenicolous fungus belongs to the Arthonia radiata group (Ihlen et al., 2004). The species was only known from Norway, Sweden and the USA (Alaska) (Ihlen et al., 2004), in Russia - from Leningrad region (Stepanchikova et al., 2013).

ARTHONIA Didyma Körb. - KvR, KzR, Ptz, VNP: 1-4. Kol! Kton! KzR! VNP!

!ARTHONIA EXCIPIENDA (Nyl.) Nyl. - KvR: right bank of the Suna River, below Kivach waterfall, $62^{\circ} 15.794^{\prime} \mathrm{N}, 33^{\circ} 58.855^{\prime} \mathrm{E}, 1,20.09 .2015$; same locality, $62^{\circ} 15.879^{\prime} \mathrm{N}, 33^{\circ} 58.730^{\prime} \mathrm{E}$, 2, 19.09.2015; left bank of the Suna River, $62^{\circ} 15.997^{\prime} \mathrm{N}, 33^{\circ} 59.685^{\prime} \mathrm{E}, 2$, 16.09.2014. VNP: bank of Vodlozero Lake near from the mouth of the Vama River, $62^{\circ} 13.026^{\prime} \mathrm{N}$, $37^{\circ} 03.456^{\prime} \mathrm{E}, 4$. 14.06.2015. Kon! Kton! KvR! VNP! This crustose lichen inhabits smooth bark of Alnus, Corylus, Berberis and Daphne spp. (Nordin et al., 2011). In Russia, the species was previously known from Mur- 
mansk region (Urbanavichus et al., 2008), Leningrad region (Kuznetsova et al., 2007) and Kaliningrad region (Dedkov et al., 2007). Distribution in Fennoscandia: Norway, Sweden, Finland (Nordin et al., 2011).

ARthonia mediella Nyl. - KvR, KzR, Ptz, VNP: 2-4. Kton! KzR!? VNP!

ARthonia Patellulata Nyl. - KvR, KzR, Ptz, VNP: 1-3. Kton! KzR! VNP!

Arthonia Radiata (Pers.) Ach. - KvR, KzR, VNP: $1-3 . \mathrm{KzR}$ !?

ARTHONIA VINOSA Leight. - KvR: 2, 3; KzR: 1, 3. KzR! RK.

Athallia PYRacea (Ach.) Arup et al. - KvR, KzR, Ptz, VNP: 1-4. KzR!

BACIDIA ARCEUTINA (Ach.) Rehm \& Arnold - KvR, KzR, Ptz: 1-3. Kol! KzR!

BACIDIA CIRCUMSPECTA (Nyl. ex Vain.) Malme - VNP: 3. Kton! VNP!

BACIDIA IGNIARII (Nyl.) Oxner - KvR, KzR, VNP: 1, 2. Kton! KvR! KzR! VNP!

BACIDIA LAUROCERASI (Del. ex Duby) Vain. - KvR, KzR, Ptz, VNP: 1-4. This species was recently reported for Karelia in provinces Kon and Kton (Tarasova \& Stepanchikova, 2016; Tarasova et al., 2016a).

BACIDIA SUBINCOMPTA (Nyl.) Arnold - KvR, KzR, Ptz, VNP: 1-4. KzR! VNP!

Biatora ALBOHYalina (Nyl.) Bagl. \& Carestia - KvR, KzR, Ptz, VNP: 1-4. Kton! KzR! VNP!

Biatora EFFloRescens (Hedl.) Räsänen - KvR, KzR, Ptz, VNP: 1-4. KzR! VNP!

BiATORA GLOBUlOSA (Flörke) Fr. - ZvR: 2, VNP: 3. Kton! VNP!

Biatora Helvola Korb ex Hellb. - KvR, KzR, Ptz, VNP: 1-4. Kol! KzR!

Biatora OCELliformis (Nyl.) Arnold - KvR, KzR, Ptz, VNP: 1-4. Kol! Kton! KzR! VNP!

Biatora Vernalis (L.) Fr. - Ptz: 1; KzR: 3.

!BIATORIDIUM MONASTERIENSE J. Lahm ex Körb. KvR: right bank of the Suna River, below Kivach waterfall, ca. $50 \mathrm{~m}$ from the river, $62^{\circ} 15.794^{\prime} \mathrm{N}, 33^{\circ} 58.855^{\prime} \mathrm{E}, 1,20.09 .2015$; right bank of the Suna River, over Kivach waterfall, central cutting line, ca. $350 \mathrm{~m}$ from river, $62^{\circ} 17.051^{\prime} \mathrm{N}, 33^{\circ} 58.271^{\prime} \mathrm{E}, 3$, 26.09.2015. Kon! KvR! The crustose lichen inhabits bark of deciduous trees Ulmus, Fraxinus, Populus spp. (Nordin et al., 2011). This species was known from different regions of central European Russia and in the southern part of North-West Russia (Urbanavichus, 2010). This locality is currently the northernmost in Russia. The nearest and previously northernmost known locality was from Leningrad region (Alexeeva \& Himelbrant, 2007; Stepanchikova et al., 2011). The distribution in Fennoscandia: Norway, Sweden and Finland (Nordin et al., 2011).

Bilimbia microcarpa (Th. Fr.) Th. Fr. - KvR, VNP: 1-4. Kton! VNP!

Bilimbia sabuletorum (Schreb.) Arnold - KvR, KzR, Ptz, VNP: 1-3. Kton! KvR! KzR! VNP!

BRYoBILIMBIA HYPNORUM (Lib.) Fryday, Printzen \& S. Ekman - VNP: 2. Kton! VNP!

BRYORIA CAPILLARIS (Ach.) Brodo \& D. Hawksw. KvR, KzR, Ptz, VNP: 2-4.

BRYORIA IMPLEXA (Hoffm.) Brodo \& D. Hawksw. KvR: 2; VNP: 4.

Bryoria NADVORniKiana (Gyeln.) Brodo \& D. Hawksw. - KzR: 3. RK.

BRYORIA VRANGiANA (Gyeln.) Brodo \& D. Hawksw. - Ptz: road to Barany Bereg village, ca. $2 \mathrm{~km}$ of Onega Lake, $61^{\circ} 50.472$ 'N $34^{\circ} 30.482^{\prime} \mathrm{E}$, 3, 22.05.2016. Det. L. Myllys. This species could be rather common in Karelia. The revision of specimens, published as $B$. fuscescens and $B$. implexa, is required due to the new data on taxonomy of $B$. implexa group (Velmala et al., 2014). The species was known in Karelia from historical (XIX century) and present collections from Petrozavodsk only (Kon, Kol) (Tarasova et al., 2013, 2015).

Buellia erubescens Arnold - KvR: 2; KzR: 1. Kon! KvR! KzR!

BuelliA DisciFormis (Fr.) Mudd - KvR: 2; VNP: $1,4$.

Caloplaca borealis (Vain.) Poelt - VNP: 3. Kton! VNP!

CAloplaca Cerina (Ehrh. ex Hedw.) Th. Fr. - KvR, KzR, Ptz, VNP: 1-4.

CANDElariella superdistans (Nyl.) Malme - VNP: 2. Kton! VNP!

CANDElariella vitellina (Ehrh.) Müll. Arg. - VNP: 1, 2. Kton! VNP!

CATinaRia NEUschildi (Körb.) P. James - KvR: 2, 3; KzR: 2; Ptz: 3. This species recently reported in Karelia in provinces Kon and Kton (Tarasova \& Stepanchikova, 2016; Tarasova et al., 2016a).

CAtinaria ATROPURPuRea (Schaer.) Vězda \& Poelt KvR, Ptz, VNP: 1-4. Kton! KvR! VNP!

Chaenotheca Brachypoda (Ach.) Tibell - KvR, KzR, Ptz, VNP: 1-3. 
Chaenotheca chlorella (Ach.) Müll. Arg. - KvR, KzR: 3. KzR!

CHAEnotheca CHRYsocephala (Turner ex Ach.) Th. Fr. - KzR: 1.

ChaEnotheca furfuracea (L.) Tibell - KvR: 3.

Chaenotheca Gracilenta (Ach.) Mattsson \& Middelb. - KvR: 2; VNP: 4. VNP! RK.

Chaenotheca hispidula (Ach.) Zahlbr. - Ptz: road to Barany Bereg villiage, ca. $2 \mathrm{~km}$ of Onega Lake, 3. It was the second finding in Karelia (Tarasova et al., 2016a). The species was previously known from the Kivach Strict Nature Reserve (Kon) (Hermansson et al., 2002).

Chaenotheca laevigata Nádv. - KvR: 2, 3; VNP: 3. Kton! VNP!

Chaenotheca stemonea (Ach.) Müll. Arg. - KvR: 2. RK.

\#Chaenothecopsis consociata (Nádv.) A. F. W. Schmidt - KzR: 1, on thalli of Chaenotheca chrysocephala. KzR!

+ChaenotheCopsis DeBilis (Sm.) Tibell - KvR: 1 . Kon! KvR! The species was only known in Karelia, from $K l$ province (Fadeeva et al., 2007).

\#ChAENOTHECOPSIS VAINIOANA (Nádv.) Tibell - KvR, KzR: 3, on thalli of Arthonia vinosa. KzR! The species was only known in Karelia from Kon province (Fadeeva et al., 2007).

Cladonia bacilliformis (Nyl.) Glück - KvR, KzR, VNP: 2, 3.

Cladonia botrytes (K. G. Hagen) Willd. - KvR: 3.

Cladonia cenotea (Ach.) Schaer. - KvR, KzR, VNP: 2-4.

Cladonia chlorophaea (Flörke ex Sommerf.) Spreng. - KvR, KzR, Ptz, VNP: 1-4. The specimens contain fumarprotocetraric acid.

Cladonia Coniocraea (Flörke) Spreng. - KvR, KzR, Ptz, VNP: 1-4.

Cladonia cornuta (L.) Hoffm. - KvR, KzR, Ptz, VNP: $1-3$.

Cladonia Cyanipes (Sommerf.) Nyl. - Ptz: 2.

Cladonia deformis (L.) Hoffm.- KvR, KzR, VNP: $2,3$.

Cladonia digitata (L.) Hoffm. - KzR, Ptz: 2, 3.

Cladonia gracilis subsp. turbinata (Ach.) Ahti - VNP: 4.

Cladonia fimbriata (L.) Fr. - KvR, KzR, Ptz, VNP: $1-4$.

Cladonia ochrochlora Flörke - KvR: 1-3; Ptz: 3; VNP: 4. Kton! KvR! VNP!

Cladonia parasitica (Hoffm.) Hoffm. - KvR: 3.

Cladonia PHYllophora Ehrh. ex Hoffm. - KvR: 3.

Cladonia Pleurota (Flörke) Schaer. - KvR: 2; KzR: 3.
Cladonia pyxidata (L.) Hoffm. - KzR: 2, 3.

Coenogonium Pineti (Ach.) Lücking \& Lumbsch - KvR: 2, 3.

COlLEMA FURFURACEUm Du Rietz - KvR, KzR, VNP: $1-3 . \mathrm{KzR}$ !

EOPYREnUla leucoplaca (Wallr.) R. C. Harris KvR: 2. KvR! The species was known in Karelia from historical collection made in $K l$ and Kon provinces (Fadeeva et al., 2007).

EVERNIA MESOMORPHA Nyl. - KzR: 1.

Evernia PRUnastri (L.) Ach. - KzR: 1; Ptz: 3; VNP: 1.

Fuscidea PUSILla Tønsberg - VNP: 1. Kton! VNP! The specimen contains divaricatic acid.

Gyalecta truncigena (Ach.) Hepp - KvR, KzR, Ptz, VNP: 1-4. Kton! KzR! VNP!

Gyalolechia FlaVorubescens (Hudson) Søchting et al. - KvR, KzR, Ptz, VNP: 1-3. Kol! Kton! VNP!

Hypogymnia PHysodes (L.) Nyl. - KvR, KzR, Ptz, VNP: $1-4$.

Hypogymina tubulosa (Schaer.) Hav. - VNP: 2, 3. LECANIA CYRTELla (Ach.) Th. Fr. - KvR: 2. KvR!

LECANIA CYRTELLINA (Nyl.) Sandst. - KvR, KzR, Ptz, VNP: 1, 2. Kton! KvR! KzR! VNP!

LeCANia dubitans (Nyl.) A. L. Sm. - KvR: 2; KzR: 1. KvR! KzR!

LeCANiA NAEGELII (Hepp) Diederich \& van den Boom - KvR, KzR, Ptz, VNP: 1-3. Kton! KvR! KzR! VNP!

LeCANORA Allophana (Ach.) Nyl. - KvR, KzR, Ptz, VNP: $1-3$.

LECANORA CARPINEA (L.) Vain. - KvR: 2; VNP: 1, 3, 4. Kon! KvR! VNP!

LECANORA CATEILEA (Ach.) A. Massal. - VNP: 4.

LECANORA CHLAROTERA Nyl. - KvR, KzR, Ptz, VNP: 1-4. Kton! KzR! VNP!

LeCANORA HAgENiI (Ach.) Ach. - KvR, KzR, Ptz, VNP: 1-4. Kton! KvR! VNP!

LeCANORA POPUlicola (DC.) Duby - KvR, KzR, VNP: 2-4. KzR!

LECANORA SAMBUCI (Pers.) Nyl. - KvR: 2. Kon! KvR!

LECANORA SYMmicta (Ach.) Ach. - Ptz: 3; VNP: 1, 4.

LecideA albofuscescens Nyl. - KvR, KzR: 3. Kon! KvR! KzR! RK.

LECIDEA BERENGeriana (A. Massal.) Nyl. - KzR: 3. KzR!

LECIDEA ERYTHROPHAEA Flörke ex Sommerf. - KvR, KzR, Ptz, VNP: 1-4. VNP!

Lecidea nylanderi (Anzi) Th. Fr. - KzR: 2; Ptz:3. $\mathrm{KzR}$ !

Lecidella elaeochroma (Ach.) M. Choisy - KvR, KzR, Ptz, VNP: 1-4. Kton! KzR! VNP! 
LEPRARIA EBURNEA J. R. Laundon - KvR, KzR: 3; Ptz: $1 . K o n !$ KvR! KzR! The specimens contain alectorialic, protocetraric and psoromic acids.

LEPRARIA ELOBATA Tønsberg - KvR: 2. Kon! KvR! The specimen contains atranorin, zeorin and stictic acid complex. The species was only known in Karelia from $K k$ province (Fadeeva et al., 2007).

LEPRARIA JACKII Tønsberg - KvR, KzR, Ptz, VNP: 1-4. Kton! Kol! KzR! VNP! The specimens contain atranorin, roccellic/angardianic, jackinic/rangiformic and norjackinic/norrangiformic acids. The species was only known in Karelia from Kk (Fadeeva et al., 2007) and Kon (Tarasova et al., 2016a, b) provinces.

LEPRARIA LOBIFICANS Nyl. - KvR: 2. KvR! The specimen contains atranorin, zeorin and stictic acid complex.

LePTOGiUm SATURninum (Dicks.) Nyl. - KvR, KzR, Ptz, VNP: 1-4.

LEPTORHAPHIS ATOMARIA (Ach.) Szatala - KvR, KzR, VNP: 2. Kton! Kon! KvR! KzR! VNP!

Lobaria PUlmonaria (L.) Hoffm. - KvR, KzR, Ptz, VNP: 1-4. RK, RR.

LOPADIUM DISCIFORME (Flot.) Kullh. - VNP: 4.

LOXOSPORA Elatina (Ach.) A. Massal. - KvR: 2; VNP: 1.

Melanelixia subaurifera (Nyl.) O. Blanco et al. KzR, Ptz: 1. RK.

Melanohalea exasperata (De Not.) O. Blanco et al. - KvR, KzR, Ptz, VNP: 1-4. KvR! VNP!

Melanohalea olivacea (L.) O. Blanco et al. - KzR, VNP: 1.

Melanohalea septentrionalis (Lynge) O. Blanco et al. - Ptz: 3.

Micarea denigrata (Fr.) Hedl. - KvR, KzR, Ptz, VNP: 1-4. Kton! VNP!

Micarea misella (Nyl.) Hedl. - KvR, KzR, VNP: 2-4. Kton! KzR! VNP!

MicAREA PRASINA Fr. - KvR, KzR: 2, 3. KzR!

Mycobilimbia CARNEOAlBida (Müll. Arg.) S. Ekman \& Printzen - KvR, KzR, Ptz, VNP: 1-4.

Mycobilimbia EPIXANTHOIDES (Nyl.) Vitik. et al. KvR, KzR, Ptz, VNP: 1-4.

Mycobilimbia tetramera (De Not.) Vitik. et al. KvR, KzR, Ptz, VNP: 1-4.VNP!

+Mycocalicium subtile (Pers.) Szatala - KvR: 2; VNP: 3. Kton! VNP!

NAetrocymbe PUnCtiformis (Pers.) R. C. Harris KvR: 1, 3. KvR!
Nephroma Bellum (Spreng.) Tuck. - KvR, KzR, VNP: 1-4. RK.

Nephroma laEvigatum Ach. - KvR: 3; VNP: 4. Kton! VNP! RK.

Nephroma Parile (Ach.) Ach. - KvR, KzR, Ptz, VNP: $1-4$.

Nephroma Resupinatum (L.) Ach. - KvR, KzR, VNP: $2-4$.

OCHROLECHIA ALBOflaVEscens (Wulfen) Zahlbr. - KvR: 1; Ptz: 1, 2. KzR! The specimens contain variolaric, lichesterinic and protolichesterinic acids.

OCHROLECHIA ARBOREA (Kreyer) Almb. - KzR: 1. KzR! The specimens contain lichexanthone, gyrophoric and lecanoric acids.

OCHROLECHIA BAHUSIENSIS H. Magn. - KvR, KzR, Ptz, VNP: 1-4.The specimens contain gyrophoric, lecanoric acids and murolic acid complex. The species recently was found in Karelia (Tarasova \& Stepanchikova, 2016; Tarasova et al., 2016a).

OCHROLECHIA MAHLUENSIS Räsänen - KvR: 1; Ptz: 3. Ptz! The specimens contain gyrophoric and lecanoric acids. The species was only known in Karelia fromVodlozero National Park (Kton) (Kukwa, 2011) and Kivach Strict Nature Reserve (Kon) (Tarasova et al., 2016b).

Ochrolechia Pallescens (L.) A. Massal. - KvR, KzR, VNP: 1-4. KzR!

OPEGRAPHA NIVEOATRA (Borrer) J. R. Laundon - KzR: 2, 3. Kon! KzR! The species was reported from Karelia without exact locality (Kopaczevskaja et al., 1977).

Opegrapha vulgata (Ach.) Ach. - KzR: 3. Kon! $\mathrm{KzR}$ !

Pachyphiale Fagicola (Arnold) Zwackh - KvR, KzR, Ptz, VNP: 1-4. Kton! VNP!

Parmelia sulcata Taylor - KvR, KzR, Ptz, VNP: $1-4$.

Parmeliella triptophylla (Ach.) Müll. Arg. - KvR, KzR, VNP: 1-3. Kton! KzR! VNP!

Parmeliopsis ambigua (Wulfen) Nyl. - KvR, KzR, Ptz, VNP: 1-4.

PARMEliopsis hyPEROPTA (Ach.) Arnold - KvR, KzR, Ptz, VNP: 1-3.

Peltigera canina (L.) Willd. - KvR, KzR, Ptz: 2, 3. Conf. O. Vitikainen.

Peltigera Degenil Gyeln. - KvR: 2. KvR! Conf. O. Vitikainen.

Peltigera leucophlebia (Nyl.) Gyeln. - KvR, KzR, Ptz: 2, 3. Conf. O. Vitikainen. 
Peltigera neckeri Hepp ex Müll. Arg. - KvR, Ptz: 1-3. Conf. O. Vitikainen.

Peltigera neopolydactyla (Gyeln.) Gyeln. - KvR: 2, 3. Conf. O. Vitikainen.

Peltigera POlydactylon (Neck.) Hoffm. - KzR: 3. Conf. O. Vitikainen.

Peltigera PRAETEXTATA (Flörke ex Sommrerf.) Zopf - KvR, KzR, Ptz, VNP: 1-4. Conf. O. Vitikainen.

Peltigera Rufescens (Weiss) Humb. - KvR: 2. Conf. O. Vitikainen.

Pertusaria Albescens (Huds.) M. Choisy \& Werner - KvR, KzR: 3. The specimens contain fatty acids.

Pertusaria Amara (Ach.) Nyl. - KvR, KzR, Ptz, VNP: $1-4$.

Pertusaria coccodes (Ach.) Nyl. - KzR: 1, 3; VNP: 4. Kon! KzR!

Pertusaria leioplaca DC. - KvR: 1, 2; VNP: 4. VNP!

+Phaeocalicium populneum (Brond. ex Duby) A. F. W. Schmidt - Ptz: road to Barany Bereg village, ca. $2 \mathrm{~km}$ of Onega lake, $61^{\circ} 50.472$ ' $\mathrm{N}$, $34^{\circ} 30.482^{\prime} \mathrm{E}, 3$. The species was known from Ks (Fadeeva et al., 2007) and Kon provinces (Tarasova et al., 2016a). RK.

Phaeophyscia ciliata (Hoffm.) Moberg - KvR, KzR, Ptz, VNP: 1-4.

Phlyctis argena (Ach.) Flot. - KvR, KzR, Ptz, VNP: $1-4$.

Physcia adscendens (Fr.) H. Olivier - KvR, KzR, VNP: 1, 2. Kton! VNP!

Physcia AIPOlia (Ehrh. ex Humb.) Fürnr. - KvR, KzR, Ptz, VNP: 1-4.

Physcia alnophila (Vain.) Loht. et al. - KvR, KzR, Ptz, VNP: 1-4.

Physcia stellaris (L.) Nyl. - VNP: 1-4.

Physconia detersa (Nyl.) Poelt - VNP: 2. Kton! VNP!

Physconia distorTa (With.) J. R. Laundon. - Ptz: 1; VNP: 1, 4. VNP!

PHYSCONIA PERISIDIOSA (Erichsen) Moberg - KzR: $1,3$.

PicCOLIA OCHROPHORA (Nyl.) Hafellner - VNP: Bostilovo, $61^{\circ} 12.001^{\prime} \mathrm{N}, 36^{\circ} 51.114^{\circ} \mathrm{E}, 1$; $61^{\circ} 11.972^{`} \mathrm{~N}, 36^{\circ} 51.009^{\circ} \mathrm{E}, 2$. Kton! VNP! The species was only known in Karelia from historical collection of the XIX century in Petrozavodsk (Tarasova et al., 2015).

Placynthiella icmalea (Ach.) Coppins \& P. James - KzR: 1.

Platismatia glauca (L.) W. L. Culb. \& C. F. Culb. - KvR, KzR, Ptz, VNP: 1-3.
Pseudoschismatomma Rufescens (Pers.) Ertz \& Tehler - KvR, KzR: 2. KzR!

Ramalina farinacea (L.) Ach. - KvR, KzR, Ptz, VNP: $1-3$.

Ramalina sinensis Jatta - KvR, KzR, Ptz, VNP: 2-4. VNP!

Ramalina thrausta (Ach.) Nyl. - KvR: 2; VNP: 4. RK.

Rinodina EXIGUa (Ach.) Gray - Ptz: 3.

RINODINA PYRINA (Ach.) Arnold - KvR: 2; VNP: 1. KvR!

RINODINA SEPTENTRIONALIS Malme - KvR: 2; KzR: 2; VNP: 4. Kton! KvR! KzR! VNP!

ROPALOSPORA VIRIDIS (Tønsberg) Tønsberg - KzR: 1; Ptz: 2.KzR! The specimens contain perlatolic acid. The species was only known from Kivach Reserve (Kon) (Hermansson et al., 2002) and Petrozavodsk (Kol) (Tarasova et al., 2016a).

Rostania occultata (Baglietto) Otálora, P. M. Jørg. \& Wedin - VNP: 4. VNP! RK.

Scoliciosporum CHLOROCOCCUM (Graewe ex Stenh.) Vězda - VNP: 2.

Scytinium subtiLE (Schrad.) Otálora et al. - KvR: 2, 3. RK.

Scytinium teretiusculum (Wallr.) Otálora et al. KvR, KzR, Ptz, VNP: 2-4. KzR!

Stenocybe PUllatula (Ach.) Stein - VNP: 1. VNP!

STRANGOSPORA MORIFORMIS (Ach.) Stein - KvR: 2, 3. KvR!

Toensbergia leucococca (R. Sant.) Bendiksby \& Timdal - Ptz: 3.

Usnea BARBATA (L.) Weber ex F. H. Wigg. - KvR: 2. Kon! KvR! RK.

USNEA DASOPOGA (Ach.) Nyl. - VNP: 3.

UsNEA SUBFLORIDANA Stirt. - KzR, VNP: 1; Ptz: 1, 3. VULPICIDA PINASTRI (Scop.) J.-E. Mattsson \& M. J. Lai - KvR, KzR, Ptz, VNP: 1-4.

XANTHORIA PARIETINA (L.) Th. Fr. - KvR, KzR, VNP: $1-3$.

\section{b. Liverworts}

Blepharostoma trichophyllum (L.) Dumort. KvR, KzR, Ptz, VNP: 1-4.

Calypogeia muelleriana (Schiffn.) Müll. Frib. KvR, KzR: 2, 3.

LEJEUNEA CAVIFOLIA (Ehrh.) Lindb. - KzR: 2. KzR! Kon! RK.

LiochlaEna Lanceolata Nees - KvR, KzR, VNP: 2-4. VNP!

LOPHOCOLEA HETEROPHYLla (Schrad.) Dumort. KvR, KzR, Ptz, VNP: 1-4. VNP! Kton! 
Lophozia gutTulata (Lindb. \& Arnell) A. Evans KzR, VNP: 3, 4. KzR! VNP! Kton!

LOPHOZIA SILvicOla H. Buch - KvR, KzR: 3. KzR! Kton!

LOPHOZIOPSIS LONGIDENS (Lindb.) Konstant. \& Vilnet - KvR, KzR: 1-3. KvR!

Neoorthocaulis atTenuatus (Mart.) L. Söderstr., De Roo \& Hedd. - KvR, KzR, Ptz, VNP: 1, 2.

Plagiochila PORELloides (Torr. ex Nees) Lindenb. - KvR, KzR, VNP: 1-4. VNP!

Ptilidium PUlChERRIMUM (Weber) Vain. - KvR, KzR, Ptz, VNP: 1-4.

Radula complanata (L.) Dumort. - KvR, KzR, Ptz, VNP: $1-4$.

\section{c. Mosses}

Abietinella ABIEtina (Hedw.) M. Fleisch. - KzR: 1. Amblystegium SeRpens (Hedw.) Schimp. - KzR: $1-3$.

BRACHYTHECIASTRUM VELUTINUM (Hedw.) Ignatov \& Huttunen - KvR: 3; Ptz: 2, 3; KzR: 3; VNP: 2. KzR! VNP!

BRACHYTHECIUM SALEBROSUM (Hoffm. ex F. Weber \& D. Mohr) Schimp. - KvR, KzR, Ptz, VNP: 1-4.

BREIDLERIA PRATENSIS (W. D. J. Koch ex Spruce) Loeske - KvR: 3; KzR: 2. KzR!

BRYUM MORAVICUM Podp. - KzR: 2. Kon! KzR!

Climacium dendroides (Hedw.) F. Weber \& D. Mohr - KvR: 3, Ptz: 3.

DicRanum montanum Hedw. - KzR: 1.

Dicranum scoparium Hedw. - KvR, KzR, Ptz, VNP: $1-4$.

EURHYNCHIASTRUM PULCHELLUM (Hedw.) Ignatov \& Huttunen - KvR: 1-3.

Fissidens adianthoides Hedw. - Ptz: 3.

HETEROClADIUM DIMORPHUM (Brid.) Schimp. - KzR: 3.

Homalia trichomanoides (Hedw.) Brid. - Ptz: 1.

Hylocomium splendens (Hedw.) Schimp. - KvR, KzR, Ptz, VNP: 1-4.

MNium STEllare Hedw. - KvR, KzR, VNP: 1-4.

Neckera Pennata Hedw. - KvR, KzR, VNP: 2; Ptz: 3. KzR! RK.

ORTHOTRICHUM OBTUSIFOLIUM Brid. - KvR 3.

ORTHOTRICHUM SPECIOSUM Nees - KvR, KzR, Ptz: 1-3; VNP: 1, 2.

Plagiomnium Cuspidatum (Hedw.) T. J. Kop. - KvR, VNP: 2, 3; KzR: 1, 2; Ptz: 1-3.

Plagiomnium DRUMmondi (Bruch \& Schimp.) T. J. Kop. - VNP: 2. Kton! VNP! RK.

Plagiothecium laetum Schimp. - KvR: 1; KzR: 3. Pleurozium schreberi (Willd. ex Brid.) Mitt. KvR, KzR: 1-3; Ptz: 2, 3; VNP: 2.
Pseudoleskeella tectorum (Funck ex Brid.) Kindb. ex Broth. - KzR: 1. KzR!

Pylaisia POlyantha (Hedw.) Schimp. - KvR, KzR, Ptz, VNP: 1-4.

Rhizomnium punctatum (Hedw.) T. J. Kop. - Ptz: 2. RHodobryum Roseum (Hedw.) Limpr. - KvR: 1, 3; KzR: 1; Ptz, VNP: 2.

RhytidiadelPhus TRIQUETRUs (Hedw.) Warnst. KvR, KzR, Ptz, VNP: 1-4.

Sanionia uncinata (Hedw.) Loeske - KvR, KzR, Ptz, VNP: 1-4.

SCIURO-HYPNUM REFLEXUM (Starke) Ignatov \& Huttunen - KzR: 3;VNP: 1.

Serpoleskea Subtile (Hedw.) Schimp. - KvR, KzR, Ptz, VNP: 1-4.

TETRAPHis PELlucida Hedw. - KzR: 3.

Thuidium Recognitum (Hedw.) Lindb. - KvR, Ptz: 2, 3; KzR: 2.

\section{RESULTS AND DISCUSSION}

A total of 222 species (excluding free-living algae and cyanobacteria) were recorded on 192 aspen trees in an area of 24 ha in the middle boreal forest communities in the Republic of Karelia, including 178 lichens and allied fungi, 32 mosses and 12 liverworts.

\section{Lichen diversity}

Species composition of lichen communities growing on aspen trees is traditionally highly diverse (Kuusinen, 1994; Pystina \& Hermansson, 1996; Ohlson et al., 1997). Study of lichens growing on aspen has greatly expanded our understanding of the biota and their distribution in the study area. Three recorded species - Arthonia biatoricola, A. excipienda, and Biatoridium monasteriense - are new for the Republic of Karelia. Seven species are new for the biogeographical province Karelia olonetsensis; 17 - for Karelia onegensis and 39 - for Karelia transonegensis. Some lichen species were found in the studied areas for the first time: two species in Petrozavodsk City, 31 - in the Kivach Strict Nature Reserve, 45 - in the Kizhi Sanctuary and 51 - in the Vodlozero National Park.

Eighteen indicator species of old-growth boreal forests were recorded (Andersson et al., 2009) - Acrocordia gemmata, Arthonia vinosa, Chaenotheca brachypoda, C. chlorella, C. gracilenta, C. laevigata, C. stemonea, Gyalecta truncigena, Leptogium saturninum, Lobaria pulmonaria, 
Nephroma bellum, N. laevigatum, $N$. parile, $N$. resupinatum, Parmeliella triptophylla, Pertusaria coccodes, Ramalina thrausta and Scytinium teretiusculum. Fifteen lichen species recorded in the studied communities associated with aspen are listed in the Red Data Book of Republic of Karelia (2007): Anaptychia ciliaris, Arthonia vinosa, Bryoria nadvornikiana, Chaenotheca gracilenta, C. stemonea, Lecidea albofuscescens, Lobaria pulmonaria, Melanelixia subaurifera, Nephroma bellum, $N$. laevigatum, Phaeocalicium populneum, Ramalina thrausta, Rostania occultata, Scytinium subtile and Usnea barbata.

Crustose lichens dominated on aspen trees (105 species), contributing $59 \%$ of the total number of lichen species. Forty-five foliose $(25 \%)$ and 28 fruticose $(16 \%)$ lichen species were recorded on aspen trees. The epiphytic cover of aspen was dominated by true epiphytes -147 species (83\%). There were also 17 muscicolous (10\%) species, the remaining $7 \%$ being represented by lignicolous (6 species), multi-substrate (5 species) and terricolous (3 species) lichens.

\section{Bryophyte diversity}

Populus tremula is characterized by supporting the highest number of epiphytic bryophyte species in Nordic countries (Hazell et al., 1998; Snäll et al., 2004) and Latvia (Mežaka et al., 2008, 2010). Most collected bryophytes were common species in Karelia. However, two species were recorded as new for the biogeographical province Karelia olonetsensis and four - for $\mathrm{Ka}$ relia transonegensis. Three liverworts (Lejeunea cavifolia, Lophozia guttulata and L. silvicola) and five bryophytes (Brachytheciastrum velutinum, Breidleria pratensis, Bryum moravicum, Neckera pennata and Pseudoleskeella tectorum) are new for the Kizhi Sanctuary. Five species of liverworts (Liochlaena lanceolata, Lophocolea heterophylla, Lophozia guttulata, L. silvicola and Plagiochila porelloides) and two moss species (Brachytheciastrum velutinum and Plagiomnium drummondit) were found for the first time in the Vodlozero National Park. In the present study, Lejeunea cavifolia was registered for the third time in Karelia. This species was collected in mixed aspen-spruce forest in the Kizhi Sanctuary. Lejeunea cavifolia is a nemoral liverwort with a predominantly circumpolar distribution, rather frequent in Russia (Konstantinova et al., 2009).
Previously, Lejeunea cavifolia was recorded in the Republic of Karelia from Karelia ladogensis (Arnell, 1956) and Paanajarvi National Park (Auer, 1944).

Eight indicators of old-growth and virgin boreal forests (Andersson et al., 2009) were recorded in our study - Lejeunea cavifolia, Liochlaena lanceolata, Neoorthocaulis attenuates, Eurhynchiastrum pulchellum, Homalia trichomanoides, Mnium stellare, Neckera pennata and Orthotrichum obtusifolium. Three bryophyte species (Neckera pennata, Plagiomnium drummondii and Lejeunea cavifolia) listed in the Red Data Book of Karelia Republic (2007) were found.

Six basic life forms (i.e. growth forms) according to K. Mägdefrau (1982) and J. Glime (2007) wefts, turfs, mats, fans, cushions and dendroids - have been found for aspen bryophytes. Furthermore, the interbryophyte life form is added to liverworts (Borovichev, 2011). In our research, most moss species belonged to wefts life form (16 species), tall turfs (4) and short turfs (3). Among the liverworts collected on aspen, the most common types of growth forms were interbryophytes (10 species) and mats (6). The greatest number of species generate several life forms adapted to different microhabitat conditions (Mägdefrau, 1982; Andrejeva, 1990; Borovichev, 2011). Amongst bryophytes recorded on aspen all substrate groups of mosses can be found epiphytic (5), epixylic (3), epigeic (6), epilytic (4), multisubstrate (14) species. The highest number of aspen liverworts was found at the base of trees represented by multisubstrate species (9); only three species were generally epiphytes (or rare epixyles).

\section{The influence of habitat on the cryptogam- ic communities associated with aspen}

The study has revealed differences in the species composition of epiphytes on aspen trunks and branches due to distinct differences in habitat conditions within a single tree. Thus, the species composition of lichens living in the lower part of the trunk and on branches (in the crown) varied significantly: 161 species were registered on the trunk, and 64 - on branches. Unlike lichens, mosses in the aspen crown were almost absent, except for the Orthotrichum species $(O$. speciosum and O. obtusifolium). 
The epiphytic cover was also quite heterogeneous in the lower part of the trunk $(0-2 \mathrm{~m})$. At the base of aspen trees $(0-25 \mathrm{~cm})$, cryptogamic cover was better developed: the mean total coverage was over $70 \%$, with mosses accounting for more than $84 \%$ of the coverage and lichens for only $16 \%$ (Table 2). The most common bryophyte species were wefts, turfs and interbryophytes, such as Hylocomium splendens, Liochlaena lanceolata, Lophozia guttulata, L. silvicola, Lophoziopsis longidens, Mnium stellare, Neoorthocaulis attenuatus, Plagiomnium cuspidatum, Plagiochila porelloides, Rhytidiadelphus triquetrus preferring tree bases and not epiphytes in a narrow sense. These species are generally epigeic, growing on soil. Few lichens were present surrounded by fast-growing large organisms (mosses and liverworts). For example, Mycobilimbia and Peltigera species possess the ability to grow on the surface of moss clumps.

At the height of $1.3 \mathrm{~m}$ above ground level, the average overall coverage is significantly lower (on average, only $38 \%$ ), with lichens contributing $61 \%$ and mosses and liverworts - 39\% (Table 2). The occurrence of true epiphytes (Lejeunea cavifolia, Neckera pennata, Orthotrichum obtusifolium, $O$. speciosum and Radula complanata) was maximum at levels higher than $1.3 \mathrm{~m}$ on the tree trunk. At a height of $1.3 \mathrm{~m}$ above ground level, the humidity might be lower than at the base of the trunk, therefore the competition between mosses and lichens may decrease and consequently the diversity and coverage of lichens increase.

Table 2. Average values of the main characteristics of epiphytes (lichens and bryophytes) on aspen tree trunks in the middle boreal forests of Republic of Karelia. Differences between groups were tested by Mann-Whitney one-way analysis of variance at $99.9 \%$ significance level $(* * *) ;{ }^{1}$ dominant taxa are species or species groups with a cover more than $1 \%$; ${ }^{2}$ treatment of the genus Mycobilimbia was according to Urbanavichus (2010); ${ }^{3}$ including Brachytheciastrum velutinum, Sanionia uncinata, and Pylaisia polyantha.

\begin{tabular}{|c|c|c|c|c|c|}
\hline \multirow{2}{*}{ Characteristics } & \multicolumn{2}{|c|}{ Height at above ground, $\mathrm{m}$} & \multirow{2}{*}{$U$-test } & \multicolumn{2}{|c|}{ Proportion of species in total cover, $\%$} \\
\hline & $0 \mathrm{~m}$ & $1.3 \mathrm{~m}$ & & $0 \mathrm{~m}$ & $1.3 \mathrm{~m}$ \\
\hline Total cover, $\%$ & $73.0 \pm 1.02$ & $37.72 \pm 0.91$ & *** & 100 & 100 \\
\hline Cover of bryophytes, $\%$ & $61.57 \pm 1.10$ & $14.75 \pm 0.66$ & $* * *$ & 84 & 39 \\
\hline Cover of lichens, $\%$ & $11.43 \pm 0.57$ & $22.97 \pm 0.76$ & $* * *$ & 16 & 61 \\
\hline Number species, no. & $4.51 \pm 0.08$ & $4.81 \pm 0.09$ & - & - & - \\
\hline Number species of bryophytes, no. & $1.65 \pm 0.05$ & $1.46 \pm 0.04$ & - & - & - \\
\hline Number species of lichens, no. & $1.85 \pm 0.06$ & $3.36 \pm 0.09$ & $* * *$ & - & - \\
\hline \multicolumn{6}{|c|}{ Cover of dominant species and groups of species ${ }^{1}, \%$ : } \\
\hline Cladonia spp. & $1.67 \pm 0.19$ & $0.42 \pm 0.09$ & $* * *$ & 2 & 1 \\
\hline Lecanora spp. & $0.02 \pm 0.01$ & $1.76 \pm 0.18$ & $* * *$ & 0.3 & 4 \\
\hline Mycobilimbia spp. ${ }^{2}$ & $4.54 \pm 0.35$ & $2.94 \pm 0.32$ & $* * *$ & 6.2 & 8 \\
\hline Nephroma spp. & $0.51 \pm 0.10$ & $0.53 \pm 0.12$ & - & 0.7 & 1 \\
\hline Peltigera spp. & $2.90 \pm 0.36$ & $0.07 \pm 0.04$ & $* * *$ & 4 & 0.2 \\
\hline Hypogymnia physodes & $<0.01$ & $1.15 \pm 0.19$ & $* * *$ & 0 & 3 \\
\hline Lobaria pulmonaria & $0.36 \pm 0.11$ & $3.59 \pm 0.34$ & $* * *$ & 0.5 & 10 \\
\hline Phlyctis argena & $0.67 \pm 0.16$ & $7.63 \pm 0.52$ & $* * *$ & 0.9 & 20 \\
\hline Brachythecium salebrosum ${ }^{3}$ & $21.19 \pm 0.97$ & $7.96 \pm 0.44$ & $* * *$ & 30 & 21 \\
\hline Radula complanata & $2.95 \pm 0.23$ & $4.18 \pm 0.36$ & - & 4 & 11 \\
\hline Hylocomium splendens & $8.16 \pm 0.69$ & $0.14 \pm 0.06$ & $* * *$ & 11 & 0.3 \\
\hline Rhytidiadelphus triquetrus & $12.92 \pm 0.97$ & $0.24 \pm 0.10$ & $* * *$ & 18 & 0.6 \\
\hline Mnium stellare & $4.63 \pm 0.51$ & $0.17 \pm 0.12$ & $* * *$ & 6 & 0.5 \\
\hline Plagiomnium cuspidatum & $4.46 \pm 0.56$ & $0.01 \pm 0.01$ & $* * *$ & 6 & 0 \\
\hline Serpoleskea subtile & $1.65 \pm 0.32$ & $0.10 \pm 0.06$ & $* * *$ & 2 & 0.3 \\
\hline
\end{tabular}



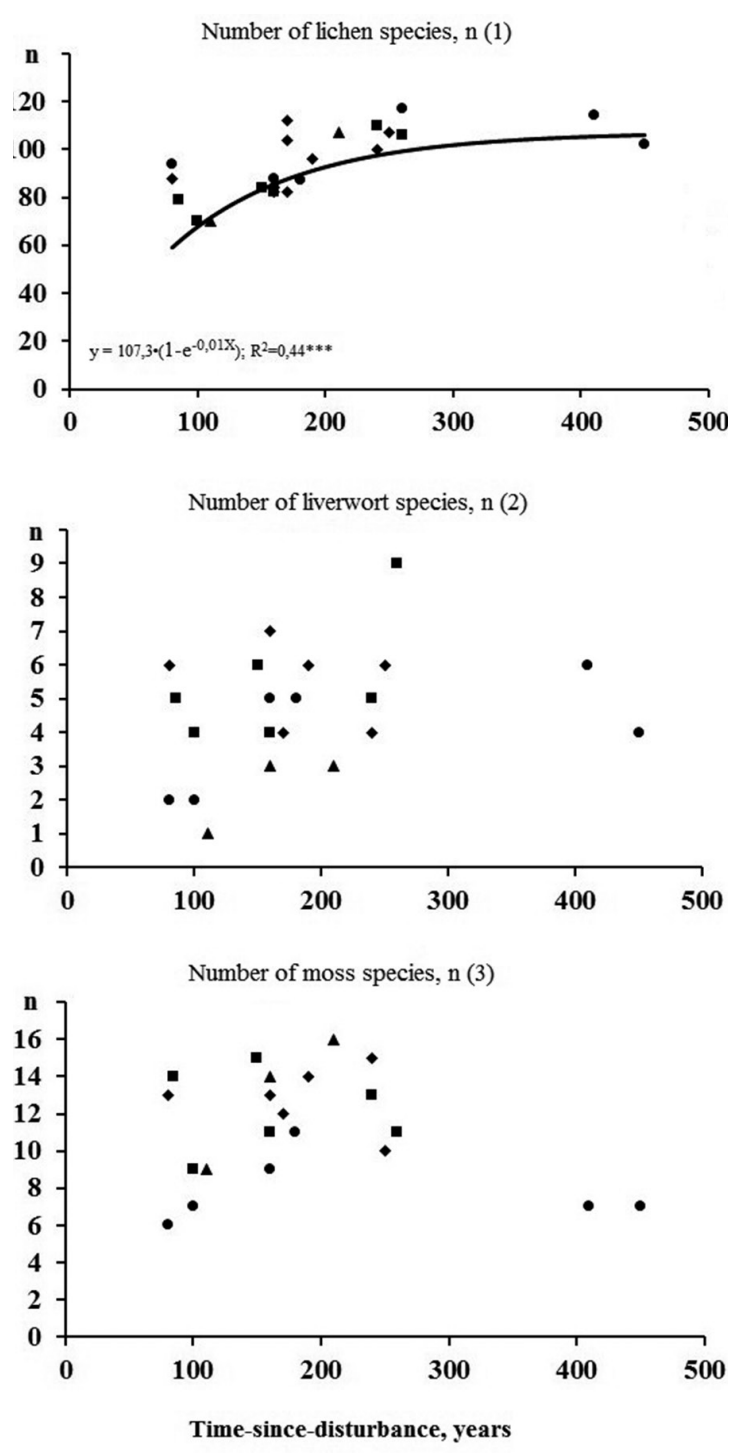

Fig. 2. The number of lichens, lichenicolous, non-lichenized fungi (1), liverwort (2) and moss (3) species on aspen per 1 ha in middle boreal forests at different time-since-disturbance (southern Karelia). The study areas are marked by the following figures: $\boldsymbol{A}$ - Petrozavodsk City, - Kizhi Sanctuary, - Vodlozero National Park, - Kivach Strict Nature Reserve. P - value of regression coefficient (R) is less than the significance level, such as $0.0011^{(* *)}$.
Lichen diversity depended on the time-sincedisturbance of the community (Fig. 2). The total number of lichens on aspen increased on average from 40 to 60 species per ha with the increase of the time since disturbance from 100 to 450 years (Fig. 2.1). Stabilization is observed at about 200 years since disturbance (Fig. 2.1). In the earlier successional stages, at 80 years since disturbance, the total lichen species diversity on aspen is richer by $10-20$ species as compared with that at 100-160 years since disturbance. Perhaps this might be due to changesfrom smooth to rough aspen bark, as well as to changes in light and moisture conditions as the proportion of spruce increases in the stand and the epiphyte community of young aspen trees changes into the epiphyte community of middleaged trees. Lichen species inhabiting early successional habitats belonged to the following genera: Buellia, Caloplaca, Evernia, Lecanora, Melanelixia, Melanohalea, Physcia, Physconia, Ochrolechia, and the species Fuscidea pusilla, Hypogymnia physodes, Leptogium saturninum, Xanthoria parietina. Late-successional species are Lobaria pulmonaria, Lopadium disciforme, Nephroma spp., Pertusaria albescens, P. coccodes, Rostania occultata, calicioid lichens (Chaenotheca brachypoda, C. chlorella, C. gracilenta, C. laevigata, C. stemonea and C. hispidula) and calicioid fungi (Chaenothecopsis vainioana and Phaeocalicium populneum). It is noteworthy that with the increase of time-since-disturbance and decrease in the number of aspen trees (Table 1) as a result of their decay in all studied forests, the lichen diversity on aspen in the communities did not decrease and remained constantly high.

For a large group of lichen species, there is no correlation between frequency of their occurrence and the time after community disturbance (see list of lichens). Typically, these were dominant and widespread species, such as Anisomeridium polypori, Bacidia subincompta, Biatora albohyalina, B. efflorescens, B. helvola, B. ocelliformis, Lecania naegelii, Lecanora allophana, Lepraria jackii, Mycobilimbia carneoalbida, $M$. epixanthoides, M. tetramera, Parmelia sulcata, Parmeliopsis ambigua, Peltigera praetextata, Phlyctis argena and Vulpicida pinastri.

No significant correlation between bryophyte diversity on aspens and the time-since-disturbance was found in our study (Fig. 2.2, 2.3). 
This result is in accordance with previous research (Mežaka \& Znotina, 2006; Mežaka et al., 2012; Putna \& Mežaka, 2014). This might arise, however, by the low number of mosses and liverworts in sample plots characterized by relatively short time after disturbance (80-100 years). Most species of aspen bryophytes were found in communities at later successional stages. The species Abietinella abietina, Bryum moravicum, Dicranum montanum and Pseudoleskeella tectorum were only found in young forests with a time from disturbance of $80-110$ years. These species are not strictly epiphytes and grow often on other substrates (Ignatov \& Ignatova, 2003, 2004). At the same time, species that are used as indicators of old-growth boreal forests, such as Homalia trichomanoides, Neckera pennata and Orthotrichum obtusifolium, were found only in communities with time after disturbance of more than 110 years.

Six liverworts - Blepharostoma trichophyllum, Lophocolea heterophylla, Lophozia guttulata, Lophoziopsis longidens, Ptilidium pulcherrimum and Radula complanata were recorded at earlier stages of succession (80-110 years). In oldgrowth forest, six liverworts were found too Liochlaena lanceolata, Lophocolea heterophylla, Lophozia guttulata, Plagiochila porelloides, Ptilidium pulcherrimum and Radula complanata.

Aspen in southern Karelia is characterized by having a high diversity of epiphytes and plays a significant role in maintaining the diversity and conservation of rare species, especially lichens. Higher humidity could create optimal conditions for the development of bryophyte cover on the aspen trunks. Therefore, lichens and mosses on the trunks of aspen often occupy different ecological niches.

\section{ACKNOWLEDGEMENTS}

We would like to express our gratitude to Roman Ignatenko, Lubov Kalacheva and Vera Androsova (Petrozavodsk State University, Russia) for their help in the field work; to Gennadii Urbanavichus (Institute of the Industrial Ecology of the North, Apatity, Russia) and Teuvo Ahti, Leena Myllys, and Orvo Vitikainen (University of Helsinki, Finland) for identification of some crustose lichens and Cladonia, Bryoria and Peltigera species; to Natalia Stavrova (Komarov Botanical Institute,
Saint-Petersburg, Russia) for consultations on determining the time-since-disturbance. We are grateful to the reviewer for valuable corrections and recommendations, which has improved the quality of our work. Warmly thanks to William Purvis (UK) for linguistic corrections. The study was supported in part by the Ministry of Education and Science of the Russian Federation (project No 5.8740.2017/k), Russian Science Foundation (project No 15-14-10023), Russian Foundation for Basic Research (research project No 15-29-02662).

\section{REFERENCES}

Abaimov, V. B. 2009. Dendrology. Textbook for students of higher schools. Academia, Moscow. 368 pp. (In Russian)

Alexeeva, N. M. \& Himelbrant, D. E. 2007. Lichens. In: Tzvelev, N. N. (executive ed.) [Volkova, E. A., Glazkova, E. A., Isachenko, G. A. \& Khramtsov, G. A., (eds)]. Environment and biological diversity of Berezovye Islands Archipelago (The Gulf of Finland). St. Petersburg, pp. 213-229. (In Russian, English summary).

Andersson, L., Alexeeva, N. \& Kuznetsova, E. (eds). 2009. Survey of biologically valuable forests in North-Western European Russia. Vol. 1. Method of survey and mapping. St.-Petersburg. 238 pp. Vol. 2. Identification manual of species to be used during survey at stand level. St. Petersburg. 258 pp. (In Russian).

Andrejeva, E. N. 1990. Air pollution impact on the moss cover of the northern taiga. In: Forest ecosystems and air pollution. Leningrad. P. 159-172. (In Russian).

Arnell, S. 1956. Hepaticae. Illustrated moss Flora of Fennoscandia 1. Lund, pp. 1-308.

Auer, A. V. 1944. Kuusamon maksasammalkasviston aineistoa. Annales Botanici Societatis "Vanamo" 21(1): 1-44.

Barkman, J. J. 1958. Phytosociology and Ecology of Cryptogamic Epiphytes. Assen, Netherlands van Gorcum Co N.Y. 628 pp.

Borovichev, E. A. 2011. Growth forms of liverworts (Marchantiophyta) of Lapland State Reserve (Murmansk Region). Vestnik of MSTU 14(3): 583-586. (In Russian).

Dedkov, V. P, Andreev, M. P. \& Petrenko, D. E. 2007. Annotated list of lichens of the Kaliningrad Region. In: Biodiversity of the Kaliningrad Region 1. Kaliningrad, pp. 79-178. (In Russian).

Degteva, S. V, Zheleznova, G. V, Pystina, T. N. \& Shubina, T. P. 2001. Coenotic and floristic structure of deciduous forests of the European North. Nauka, St. Petersburg. 269 pp. (In Russian).

Dyrenkov, S. A. 1984. Structure and dynamics of boreal spruce forests. Science. Leningrad. 176 pp. (In Russian). 
Ellis, C. J. \& Coppins, B. J. 2007. Reproductive strategy and the compositional dynamics of crustose lichen communities on aspen (Populus tremula L.) in Scotland. Lichenologist 39: 377-391. https:/ / doi.org/10.1017/S0024282907006937

Esseen, P.-A., Renhorn, K.-E. \& Pettersson, R. B. 1996. Epiphytic lichen biomass in manage and old growth boreal forests: effect of branch quality. Ecological Applications 6: 228-238. https://doi. org/ $10.2307 / 2269566$

Fadeeva, M. A., Golubkova, N. S., Vitikainen, O. \& Ahti, T. 2007. Conspectus of lichens and lichenicolous fungi of the Republic of Karelia. Petrozavodsk. 194 pp. (In Russian).

Fadeeva, M. A., Manninen, O. \& Syrjänen, K. 2014. List of lichens and allied fungi collected on Zaonezhye Peninsula. Biogeography, landscapes, ecosystems and species of Zaonezhye Peninsula, in Onega Lake, Russian Karelia. Reports of the Finnish Environment Institute 40: 207-222.

Fritz, Ö., Gustafsson, L. \& Larsson, K. 2008. Does forest continuity matter in conservation? - A study of epiphytic lichens and bryophytes in beech forests of Southern Sweden. Biological Conservation 141 (3): 655-668. https://doi.org/10.1016/j. biocon.2007.12.006

Gauslaa, Y. 1995. The Lobarion, an epiphytic community of ancient forests threatened by acid rain. $\mathrm{Li}$ chenologist 27: 59-76. https://doi.org/10.1017/ S0024282995000077

Gjerde, I., Blom, H. H., Lindblom, L., Sætersdal, M. \& Høistad Schei, F. 2012. Community assembly in epiphytic lichens in early stages of colonization. Ecology 93: 749-759. https://doi. org/10.1890/11-1018.1

Glime, J. M. 2007. Bryophyte Ecology. Vol. 1. Ebook sponsored by Michigan Technological University and the International Association of Bryologists. Ver. 15 August 2013 - http: / / www.bryoecol.mtu. edu (25 March 2017).

Gustafsson, L. \& Eriksson, I. 1995. Factors of importance for the epiphytic vegetation of aspen Populus tremula with special emphasis on bark chemistry and soil chemistry. Journal of Applied Ecology. 32: 412-424. https: / / doi.org/10.2307/2405107

Hazell, P., Kellner, O., Rydin, H. \& Gustafsson, L. 1998. Presence and abundance of four epiphytic bryophytes in relation to density of aspen Populus tremula and other stand characteristics. Forest Ecology and Management 107: 147-158. https:/ / doi.org/10.1016/S0378-1127(97)00330-7

Hedenås, H. \& Eriksson, L. 2000. Epiphytic macrolichens as conservation indicators: successional sequence in Populus tremula stands. Biological Conservation, 93: 43-53. https://doi.org/10.1016/ S0006-3207(99)00113-5

Hermansson, J., Tarasova, V. N., Stepanova, V. I. \& Sonina, A. V. 2002. Lichens of Kivach Reserve. Flora and fauna of reserves 101: 1-34. (In Russian).
Hill, M. O., Bell, N., Bruggeman-Nannenga, M. A., Brugués, M., Cano, M. J., Enroth, J., Flatberg, K. I., Frahm, J.-P., Gallego, M. T., Garilleti, R., Guerra, J., Hedenäs, L., Holyoak, D. T., Hyvönen, J., Ignatov, M. S., Lara, F., Mazimpaka, V., Munõz, J. \& Söderström, L. 2006. An annotated checklist of the mosses of Europe and Macaronesia. Journal of Bryology 28: 198-267. https://doi. org/10.1179/174328206X119998

Ignatov, M.S., Ignatova, E.A. 2003. Moss flora of the Middle European Russia. Vol. 1: Sphagnaceae Hedwigiaceae. Moscow, KMK Scientific Press Ltd. 608 pp. (In Russian).

Ignatov, M. S., Ignatova, E. A. 2004. Moss flora of the Middle European Russia. Vol. 2: FontinalaceaeAmblystegiaceae. Moscow, KMK Scientific Press Ltd. p. 335 pp. (In Russian).

Ignatov, M. S., Afonina, O. M. \& Ignatova, E. A. et al. 2006. Check-list of mosses of East Europe and North Asia. Arctoa 15: 1-130. https://doi. org/10.15298/arctoa.15.01

Ihlen, P. G., Owe-Larsson, B. \& Tønsberg T. 2004. Arthonia biatoricola sp. nov. from north-western Europe and northern Pacific North America. Symbolae Botanicae Upsaliensis 34 (1): 107-111.

Ipatov, V. S. 1960. Aspen forests types of the Northwest of the RSFSR. Bulletin of the Leningrad State University 3 (1): 23-40. (In Russian).

John, E. \& Dale M. R. T. 1995. Neighbor relations within a community of epiphytic lichens and bryophytes. Bryologist 98: 29-37. https://doi. org/ 10.2307/3243637

Kazimirov, N. I. 1971. Spruce forests of Karelia. Leningrad. 139 pp. (In Russian).

Konstantinova, N. A., Bakalin, V. A., Andreeva, E. N., Bezgodov, A. G., Borovichev, E. A., Dulin, M. V. \& Mamontov Yu. S. 2009. Checklist of liverworts (Marchantiophyta) of Russia. Arctoa 18: 1-63. https://doi.org/10.15298/arctoa.18.01

Kopaczevskaja, E. G., Makarevicz, M. F. \& Oxner A. N. 1977. Handbook of the lichens of the U.S.S.R. 4. Verrucariaceae - Pilocarpaceae. Leningrad. 344 pp. (in Russian).

Koskinen, A. 1955. Über die Kryptogamen der Bäume, besonders die Flechten, im Gewässergebiet des Päijänne sowie an den Flüssen Kalajoki, Lestijoki und Pyhäjoki. Florstische, soziologische und ökologische Studie I., Helsinki. 176 pp.

Kukwa, M. 2011. The lichen genus Ochrolechia in Europe. Gdańsk. 309 pp.

Kuusinen, M. 1994. Epiphytic lichen flora and diversity on Populus tremula in old-growth and managed forests of southern and middle boreal Finland. Annales Botanici Fennici 31: 245-260.

Kuusinen, M. 1996. Cyanobacterial macrolichens on Populus tremula as indicators of forest continuity in Finland. Biological Conservation 75: 43-49. https://doi.org/10.1016/0006-3207(95)00039-9

Kuusinen, M. \& Sitonen, J. 1998. Epiphytic lichen diversity in old-growth and managed Picea 
abies stands in southern Finland. Journal of Vegetation Science 9: 283-292. https://doi. org/10.2307/3237127

Kuznetsova, E., Ahti, T. \& Himelbrant, D. 2007. Lichens and allied fungi of the Eastern Leningrad Region. Norrlinia 16: 1-62.

Lesica, P., McCune, B., Cooper, S. V. \& Hong, W. S. 1991. Differences in lichen and bryophyte communities between old-growth and managed second-growth forests in the Swan Valley, Montana. Canadian Journal of Botany 69: 1745-1755. https:/ / doi.org/10.1139/b91-222

Lõhmus, P. 2003. Composition and substrata of forest lichens in Estonia: a meta-analysis. Folia Cryptogamica Estonica 40: 19-38.

Lõhmus, A., Lõhmus, P. \& Vellak, K. 2007. Substratum diversity explains landscape-scale covariatin in the species-richness of bryophytes and lichens. Biological Conservation 135: 405-414. https:// doi.org/10.1016/j.biocon.2006.10.015

Mägdefrau, K. 1982. Life-forms of bryophytes. Bryophyte Ecology. London, pp. 45-58. https://doi. org/10.1007/978-94-009-5891-3_2

McCune, B. 2000. Lichen communities as indicators of forest health. Bryologist 103: 353-356. https:/ / doi.org/10.1639/0007-2745(2000)103[0353:LCA IOF]2.0.CO;2

Mežaka, A. \& Znotina, V. 2006. Epiphytic bryophytes in old growth forests of slopes, screes and ravines in north-west Latvia. Acta Universitatis Latviensis 710: 103-116.

Mežaka, A., Brūmelis, G. \& Piterāns, A. 2008. The distribution of epiphytic bryophyte and lichen species in relation to phorophyte substrate in Latvian natural old-growth broad leaved forests. Folia Cryptogamica Estonica 44: 89-99.

Mežaka, A., Brūmelis, G. \& Piterāns, A. 2010. Epiphytic bryophyte and lichen communities in relation to tree and forest stand variables in Populus tremula forests of south-east Latvia. Acta Biologica Universitatis Daugavpiliensis 2: 1-8.

Mežaka, A., Brūmelis, G. \& Piterāns, A. 2012. Tree and stand scale factors affecting richness and composition of epiphytic bryophytes and lichens in deciduous woodland key habitats. Biodiversity and Conservation 21 (12): 3221-3241. https:// doi.org/10.1007/s10531-012-0361-8

Mikhailova, I., Trubina, M., Vorobeichik, E. \& Scheidegger, C. 2005. Influence of environmental factors on the local-scale distribution of cyanobacterial lichens: case study in the North Urals, Russia. Folia Cryptogamica Estonica 41: 45-54.

Molchanov, A. A. 1961. Forest and climate. Moscow. 279 pp. (In Russian).

Morozova, R. M. 1991. Forest soils of Karelia. Leningrad. 184 pp. (In Russian).

Nazarova, L. E. 2003. Climate. Biodiversity of biota of Karelia: conditions offormation, communities, species. Petrozavodsk. The Karelia Scientific Centre of RAS. P. 6-8. (In Russian).
Nordin, A., Moberg, R., Tønsberg, T., Vitikainen, O., Dalsätt, Å., Myrdal, M., Snitting, D. \& Ekman, S. 2011. Santesson's Checklist of Fennoscandian Lichen-forming and Lichenicolous Fungi. Ver. April 29, 2011 - http://130.238.83.220/santesson/ home.php (25 March 2017).

Norrlin, J. P. 1876. Flora Kareliae Onegensis. II. Lichens. Meddelanden af Societas pro Fauna et Flora Fennica 1: 1-42.

Ohlson, M., Söderström, L., Hörnberg, G., Zackrisson, O. \& Hermansson, J. 1997. Habitat qualities versus long-term continuity as determinants of biodiversity in boreal old-growth swamp forests. Biological Conservation 81: 221-231. https:/ / doi. org/10.1016/S0006-3207(97)00001-3

Ojala, E., Mönkkönen, M. \& Inkeröinen, J. 2000. Epiphytic bryophytes on European aspen Populus tremula in old-growth forests in NorthEastern Finland and in adjacent sites in Russia. Canadian Journal of Botany 78 (4): 529-536. https://doi. org/10.1139/b00-023

Orange, A., James, P. W. \& White, F. J. 2001. Microchemical methods for the identification of lichens. London. $101 \mathrm{pp}$.

Putna S. \& Mežaka A. 2014. Preferences of epiphytic bryophytes for forest stand and substrate in North-East Latvia Folia Cryptogamica Estonica 51: 75-83. https:/ / doi.org/10.12697/fce.2014.51.08

Pykälä, J., Heikkinen, R.K., Toivonen, H. \& Jääskeläinen, K. 2006. Importance of forest act habitats for epiphytic lichens in Finnish managed forests. Forest ecology and management 223: 84-92. https://doi.org/10.1016/j.foreco.2005.10.059

Pystina, T. N. \& Hermansson, J. 1996. Epiphytic lichens on trunks of Populus tremula L. in middle boreal aspen forest. Biological diversity of anthropogenically transformed landscapes in the northeast of European Russia. Proceedings of the Komi Scientific Centre of RAS 149: 109-119. (In Russian).

Red Data Book of Karelian Republic. 2007. Petrozavodsk. 368 pp. (In Russian).

Red Data Book of Russian Federation (plants and fungi). 2008. Moscow. 885 pp. (In Russian).

Richardson, D. H. S. 1992. Pollution monitoring with lichens. Naturalists' Handbooks 19: 1-76.

Shubina, T. P., Zheleznova, G. V., Degteva, S. V. \& Kustysheva, A. A. 1996. Bryophytes of middle boreal aspen forests (Komi Republic). Biological diversity of anthropogenically transformed landscapes in the northeast of European Russia. Proceedings of the Komi Scientific Centre of RAS 149: 102-108. (In Russian).

Snäll, T., Hagström, A., Rudolphi, J. \& Rydin, H. 2004. Distribution pattern of the epiphyte Neckera pennata on three spatial scales - importance of past landscape structure, connectivity and local conditions. Ecography 27: 757-766. https:/ / doi. org/10.1111/j.0906-7590.2004.04026.x 
Söderström, L., Hagborg, A., von Konrat, M., Bartholomew-Began, S., Bell, D., Briscoe, L., Brown, E., Cargill, D. C., Costa, D. P., Crandall-Stotler, B. J., Cooper, E. D., Dauphin, G., Engel, J. J., Feldberg, K., Glenny, D., Gradstein, S. R., He, X., Heinrichs, J., Hentschel, J., Ilkiu-Borges, A. L., Katagiri, T., Konstantinova, N. A., Larraín, J., Long, D. G., Nebel, M., Pócs, T., Felisa, P. F., Reiner-Drehwald, E., Renner, M. A. M., Sass-Gyarmati, A., SchäferVerwimp, A., Moragues, J. G. S., Stotler, R. E., Sukkharak, P., Thiers, B. M., Uribe, J., Váňa, J., Villarreal, J. C., Wigginton, M., Zhang, L. \& Zhu, R.-L. 2016. World checklist of hornworts and liverworts. PhytoKeys 59: 1-828. https://doi. org/10.3897/phytokeys.59.6261

Stavrova, N. I., Gorshkov, V. V. \& Katyutin, P. N. 2016. Structure formation of forest tree species coenopopulations during post-fire recovery of northern taiga forests. Proceedings of the Karelian Scientific Centre of RAS 3: 10_28. (In Russian).

Stepanchikova, I. S., Himelbrant, D. E., Kukwa, M. \& Kuznetsova, E. S. 2011. New records of lichens and allied fungi from the Leningrad Region, Russia II. Folia Cryptogamica Estonica 48: 85-94.

Stepanchikova, I. S., Tagirdzhanova, G. M. \& Himelbrant, D. E. 2013. The lichens and allied fungi of the Smorodinka River valley (Leningrad region). Novitates systematicae planetarium non vascularum 47: 262-278.

Strazdina, L. 2010. Bryophyte community composition on an island of Lake Cieceres, Latvia: dependence on forest stand and substrate properties. Environmental and Experimental Biology 8: 49-58.

Tarasova, V. N., Androsova, V. I., Sonina, A. V. \& Ahti, T. 2015. The lichens from the City of Petrozavodsk in the Herbarium of the Botanical Museum, University of Helsinki. Folia Cryptogamica Estonica 52: 41-50. https://doi.org/10.12697/ fce.2015.52.06
Tarasova, V. N., Androsova, V. I., Stepanchikova, I. S. \& Sonina, A. V. 2016a. New records of lichens for the territory of Petrozavodsk. Modern problems of science and education, 5. URL: http://www. science-education.ru/article/view?id=25411 (In Russian).

Tarasova, V. N., Kalacheva, L. A., Shvetzova, V. O., Stepanchikova, I. S. \& Ahti T. 2016b. Lichens on Scots pine (Pinus sylvestris) in green-moss pine forests of Kivach Reserve. Proceedings of the Kivach Reserve 7: 3-12. (In Russian).

Tarasova, V. N., Sonina, A. V., Androsova, V. I. \& Ahti, T. 2013. The present lichen flora of the city of Petrozavodsk. Folia Cryptogamica Estonica 50: 57-66. https:/ / doi.org/10.12697/fce.2013.50.08

Tarasova, V. N. \& Stepanchikova I. S. 2016. New lichens to Karelia Republic. Proceedings of Petrozavodsk State University. Biological sciences 4 (157): 78-82. (In Russian).

Uliczka, H. \& Angelstam, P. 1999. Occurrence of epiphytic macrolichens in relation to tree species and age in managed boreal forest. Ecography 22: 396405. https://doi.org/10.1111/j.1600-0587.1999. tb00576.x

Urbanavichus, G., Ahti T. \& Urbanavichene, I. 2008. Catalogue of lichens and allied fungi of Murmansk Region, Russia. Norrlinia 17: 1-80.

Urbanavichus G. P. 2010. A checklist of the lichen flora of Russia. St. Petersburg. 194 pp. (In Russian).

Velmala, S., Myllys, L., Goward, T., Holien, H. \& Halonen, P. 2014. Taxonomy of Bryoria section Implexae (Parmeliaceae, Lecanoromycetes) in North America and Europe, based on chemical, morphological and molecular data. Annales Botanici Fennici 51(6): 345-371. https://doi. org/10.5735/085.051.0601

Volkov, A. D. 2008. Forest types of Karelia. Petrozavodsk. 180 pp. (In Russian). 
142 Folia Cryptog. Estonica 